\title{
HOW TO USE THE OVIPOSITOR DRILLING MECHANISM OF HYMENOPTERA FOR DEVELOPING A SURGICAL INSTRUMENT IN BIOMIMETIC DESIGN
}

\author{
K. NAKAJIMA \& O. SCHWARZ \\ Department of Biomechatronic Systems, Fraunhofer Institute for Manufacturing Engineering and Automation, Germany.
}

\begin{abstract}
Hymenopterous insects are able to drill several inches into fresh wood with an egg-laying organ (ovipositor) as thin as a hair, to deposit their eggs. Up to now only one method of boring, i.e. rotary drilling, is technically used. The transfer of biomimetic principle to the field of orthopedic surgery was done in the bionic development process. The analysis of the anatomy and physiology of hymenoptera led to the realization that it is of utmost importance to have the drill composed of three parts to enable a balanced drilling process without transfer of torque to the work piece. This principle was then implemented in a prototypical functional and design model of a drill rasp for creating cavities in the thigh bones for the form-fitted insertion of cementless hip prosthesis stems. The challenge was to design a drilling device for application in an operating theater. Thus, the emphasis was on an ergonomic user experience, hygiene before and during the process, the specific anatomy of the thigh bone, and an optimal workflow during the operation. A patent application was filed for the technical implementation and received the brand name Sirex ${ }^{\mathrm{TM}}$ in accordance with a wood wasp genus.
\end{abstract}

Keywords: bionic design, boring mechanism, hip prosthesis, reciprocating drilling, surgical instrument.

\section{INTRODUCTION}

Surgical instruments are designed to optimally fulfill their intended function. Thus, a much wider biomimetic approach can be chosen, which takes into account all varieties of life forms and uses them as inspiration. Due to the similarities in their functions and constraints, it is easily possible to adapt concepts from animal mouthpieces and gripping mechanisms for medical engineering challenges. Following the seven steps of biomimetic design by Rechenberg [1], this is especially straightforward for surgical instruments.

\section{DEVELOPING PROCESS}

\subsection{Identification of a problem in medical science/in medical engineering}

Hence, appropriate use cases in medical engineering with a focus on drilling had to be found. Interviews with experts and a market study led to the conclusion that hip surgery would provide a good scenario, as cavities have to be drilled into the thigh bone. After choosing the use case, the 'topdown-approach in biomimetics' as defined by the VDI (Verein Deutscher Ingenieure) in guideline 6220 was applied (see Fig. 1). The aim was to find a technical solution by first defining a concrete technical problem, which may be solvable by drilling mechanisms found in nature. The medical requirements were defined by interviews with experts and the German Medical Device Directive. Further analyses of the operational workflow from live observations during operations were incorporated into the requirements. The requirements were further optimized during the entire design from concept phase to prototyping process. 


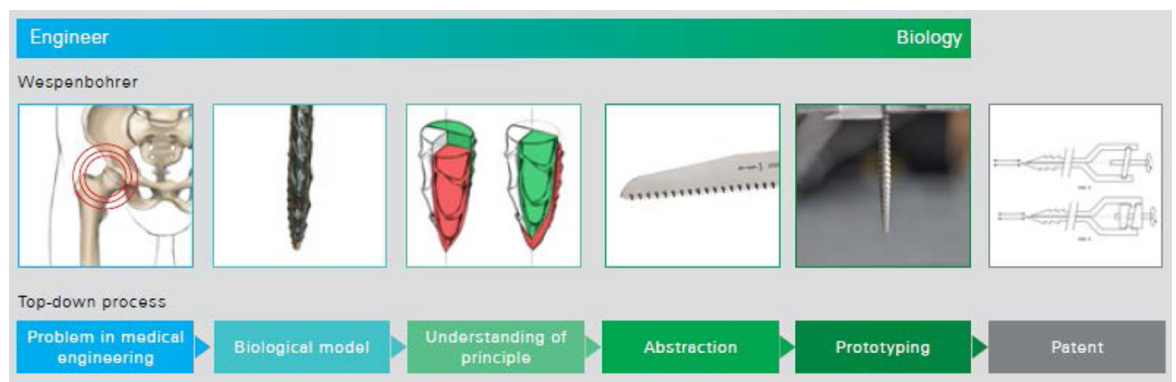

Figure 1: Top-down approach applied to the design process of 'Sirex ${ }^{\circledR}$ (based on Plant Biomechanics Group Freiburg 2010, VDI 6220).

\subsection{Analysis of biological models}

In the first step, the taxonomic classification in the order of Hymenoptera was examined and limited to two groups of particular interest, i.e. Symphyta (sawflies) and Apocrita (insects with a narrow 'waist') [2]. A part of the first group is the suborder of wood wasps (Siricidae), with approximately 100 species, which prefer coniferous wood. To the second group belongs the family of ichneumon wasps, which also drills into wood but with one major difference: They go for the larvae living in the wood, into which they lay their eggs. The aim of the investigation was to learn how some species live and how their sting works. The Giant Woodwasp (Urocerusgigas) drills a 10- to 15-mm hole into the wood. The mechanism applied by these species of wood and ichneumon wasps is the so-called reciprocating principle. The ovipositor of the wood wasp is made of chitin and consists of an upper ovipositor valve and two lower ovipositor valves with multiple saw blades at the top. The upper ovipositor valve has two cavities (channels) that ensure stability and protect the valve from bending over.

The lower ovipositor valves are flexibly bound to the upper ovipositor valve by the so-called sliding ribs (olistheter). The lower ovipositor valves accommodate a duct for transporting the eggs into the hole. The tip serves for twisting and screwing in, the section in-between is necessary for expanding the passage. The rest is equipped with prongs facing backwards and serves for anchoring the now cylindrical ovipositor. The drill of a wood wasp withstands even the toughest use without breaking (Fig. 2).

\subsection{Understanding of principle}

The up and down motion provides the friction and planes the wood piece by piece. The two drill tips are always working alternately upwards or downwards, so that one tip always remains in the hole and provides counterforce (see Fig. 3). This makes it possible to drill precisely. This principle operates without large axial force application and works like a rasp and unlike a screw or a spiral drill.

\subsection{Abstraction}

The theorem of the prohibition of slavish imitation of the natural design in the biomimetic transfer was followed. Division of the drill into three equally sized parts instead of two quarter circles and one half circle. The teeth of the drill should be aligned inconsistently, changing from proximal to 


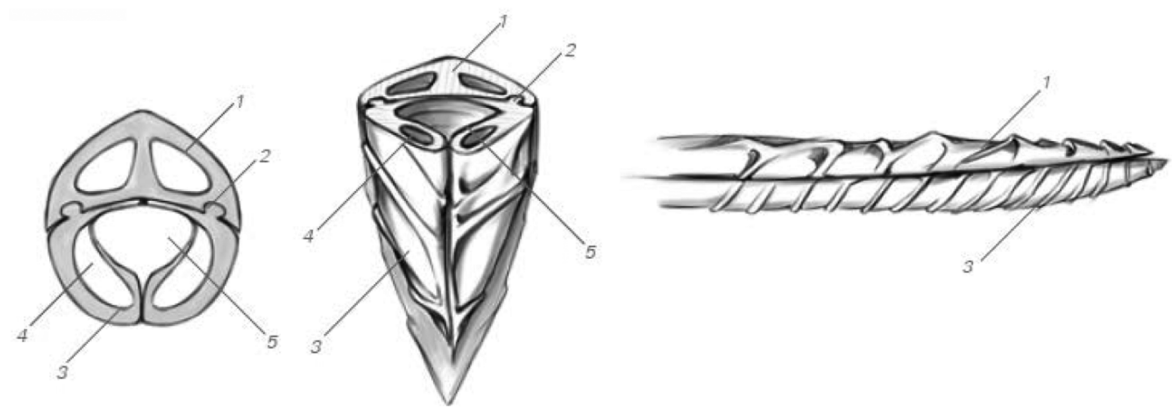

Figure 2: Structure of the ovipositor. Schematic representation in a longitudinal cut: (1) Upper ovipositor valve with two channels, (2) sliding ribs for the lower ovipositor valves, (3) lower ovipositor valves, (4) lower ovipositor valve tubules and (5) duct for discharging eggs.
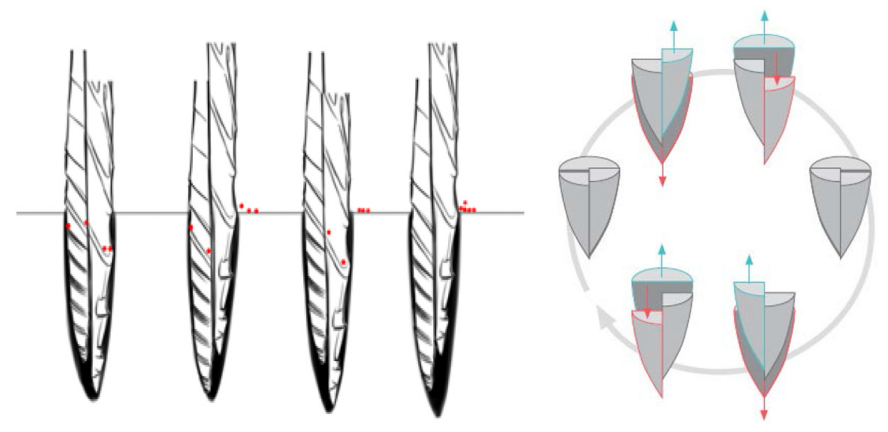

Figure 3: Simplified illustration of the reciprocating motion (left); observed motion of the three-part drill (right).

distal. The only other two applications of a biologically inspired penetration system are used for astrobiological studies and percutaneous interventions. Both are using a two-part drill bit $[3,4]$.

To analyze the motion of the three drill tips, the movement was converted into a curve graph. As shown in Fig. 4, each color represents a drill tip, resulting in a sine curve. The violet-colored curve is continuous and uniform, which means the drill tip is constantly moving up and down. The two other curves in green and red are interrupted, which means they are alternately moving up and down.

\subsection{Prototyping}

Before developing the bionic drilling instrument, we tried to obtain all possible requirements influencing the design of the femur drill.

\subsubsection{Requirements for medical products}

An instrument used for operating on humans poses a high risk to health. This is why a medical product must meet very strict requirements. It has to comply with many standards and statutory provisions. 

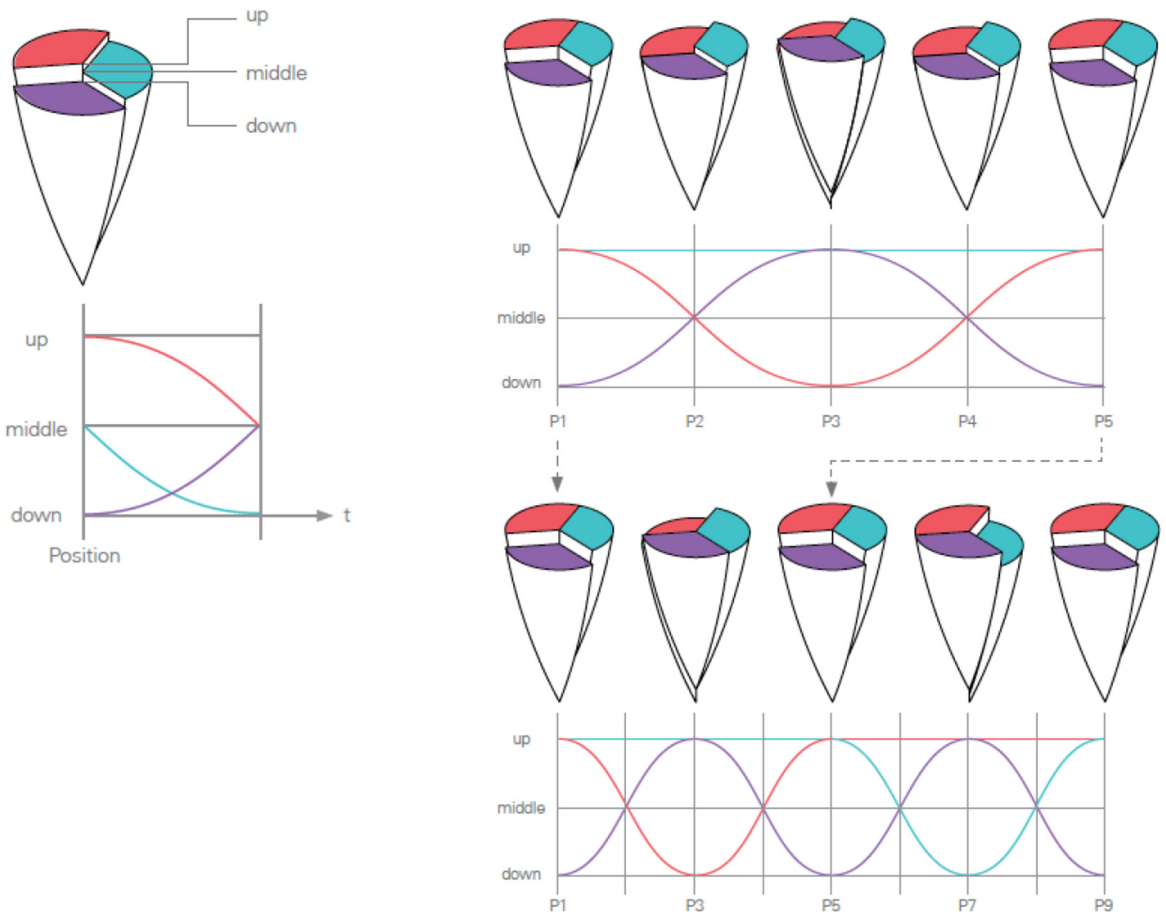

Figure 4: Motion pattern of the three drill tips.

The most important standards, certificates and categories are listed below.

- CE marking: EU Regulation 765/2008; EN ISO 13485 'Medical devices - Quality management systems'.

- Medical Devices Act (Medizinproduktegesetze, MPG): The European Directive for Medical Products 93/42 EWG.

- American FDA (Food and Drug Administration).

- Other important ISO standards: EN 30993; ISO 10993 (material), ISO 10993 1-20 (biocompatibility).

\subsubsection{Requirements of stakeholders}

Before a hospital invests in a new instrument, before a medical product can ever find a market, the demands of different groups of people in terms of design and functionality have to be met. Figure 5 shows the requirements of all possible stakeholders.

\subsubsection{Requirements for the subassemblies}

The requirements in Fig. 5 will now be included in requirement specifications for component design and materials to be used.

2.5.3 1 Rasp design The size of the rasp depends on the type of prosthesis. There is no pre-defined size for a hip prosthesis (see Fig. 6a). Each manufacturer has different prosthesis systems. In general, 


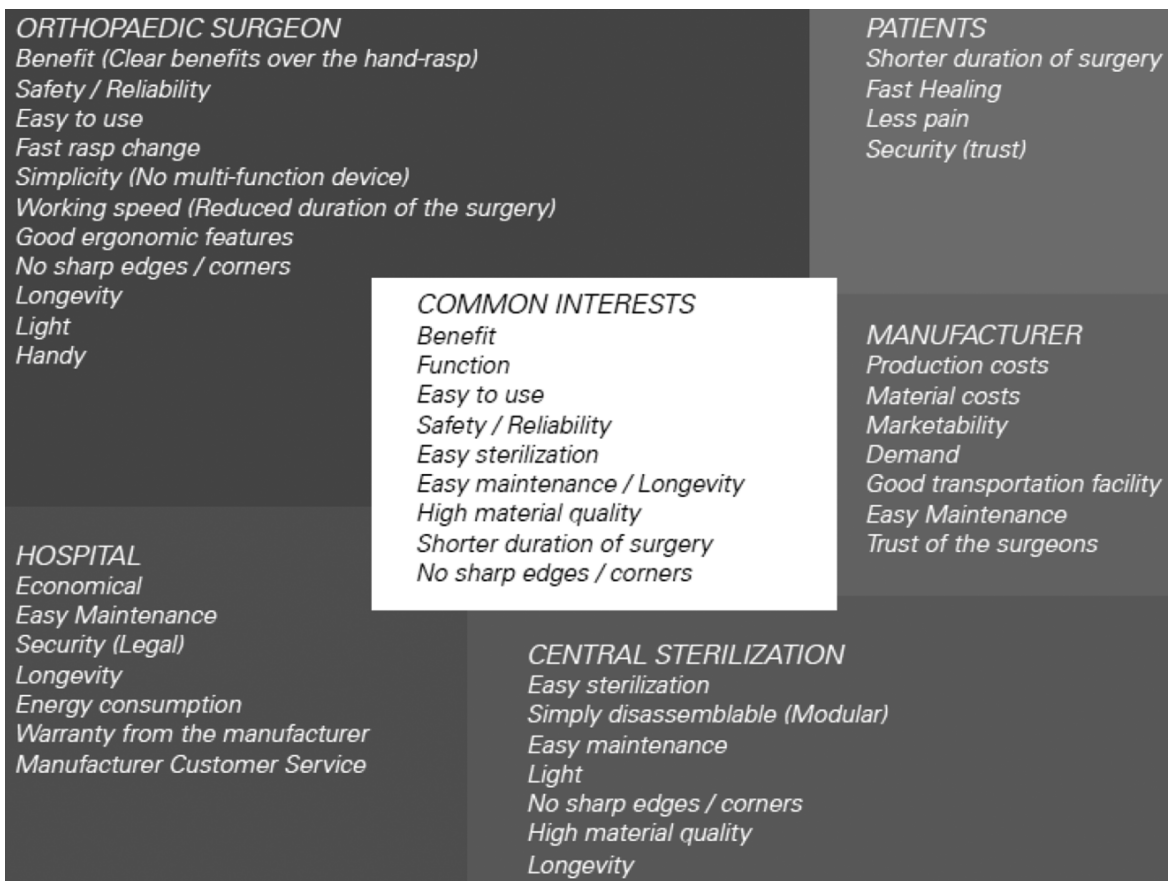

Figure 5: Requirements of stakeholders.

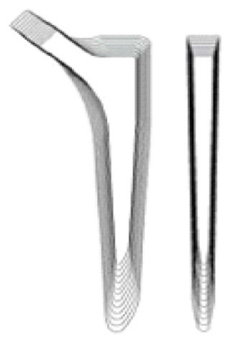

a)

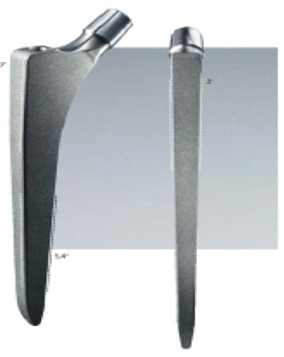

b)

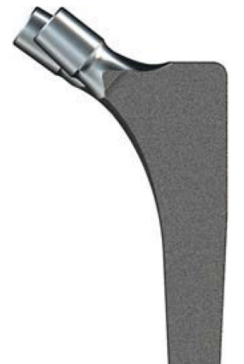

c)

Figure 6: (a) Different sizes of a hip prostheses, (b) cementless hip replacement prosthesis shaft Aesculap ${ }^{\circledR} \mathrm{TRJ}^{\circledR}$ (source: B. Braunc); the two different centrum-collum-diaphysis angles (CCD): $123^{\circ}$ and $131^{\circ}$.

they are offered in different sizes. Accordingly, it has to be decided which prosthesis system to use to be able to adapt the size and shape of the rasps.

There are three basic versions of THRPs (total hip replacement prostheses):

- Cementless prosthesis.

- Cemented prosthesis.

- Hybrid prosthesis. 
With all three versions, the femur is prepared with a rasp. A precise cavity is only required for the cementless prosthesis (and to some extent for the hybrid version, too), while in the cemented version the cement is used to fill up a larger cavity. This method is most often used in elderly patients, but is applied less frequently now; it accounts for $<20 \%$ [5].

The cementless prosthesis must fit exactly without leaving any space between the prosthesis and the bone. Too much pressure or too much space around the stem can bring about the dreaded prosthetic loosening.

Thus, it was decided to focus on cementless prostheses, and the Aesculap long stem-prosthesis system SPC stem 12/14 was selected (see Fig. 6b). It has 12 different length options on offer, each with two angle versions for angles between the neck and the shaft of the thigh bone (see Fig. 6c). Accordingly, the length of the stem varies between 145.6 and $189.6 \mathrm{~mm}$.

The lessons learned from the first functional model help with the design of the rasp. Particularly important are the shape and the alignment of the saw teeth (rasp teeth).

Saw teeth

The aim in designing the saw teeth is to develop 'self-drilling' features for the rasp. Thus, the alignment of the saw teeth is proximal. Cutting angle $\alpha$ : $80-90^{\circ}$ and wedge angle $\beta$ : $60^{\circ}$.

Tip angle

The tip angle is set at $30^{\circ}$, based on experiments with the functional model.

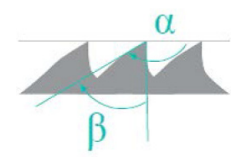

\section{Olistheter/dovetail guide}

In line with the biological model, the so-called olistheters need not be attached along the entire length of the rasp. Their function is to connect the tips. To be on the safe side, they should be linked to each other at another point.

\section{Basic form}

The basic form should be tailored to the THRP long stem model SPC stem CCD $135^{\circ} 12 / 14$. The specimen is in size 5 , to which the drill is adapted.

\section{Uniform distribution}

The cross-section of the head of the prosthesis stem is squareshaped. Iit is uniformly distributed over a $120^{\circ}$ angle, with the bottom third being slightly smaller than the two upper thirds. This is compensated by choosing a smaller angle of $110^{\circ}$ for the upper two parts.

Safety edge

Although the rasp is 'self-digging', it must not 'dig itself' too deeply. To prevent this from happening, it should be furnished with an edge that prevents 'digging too deep'.
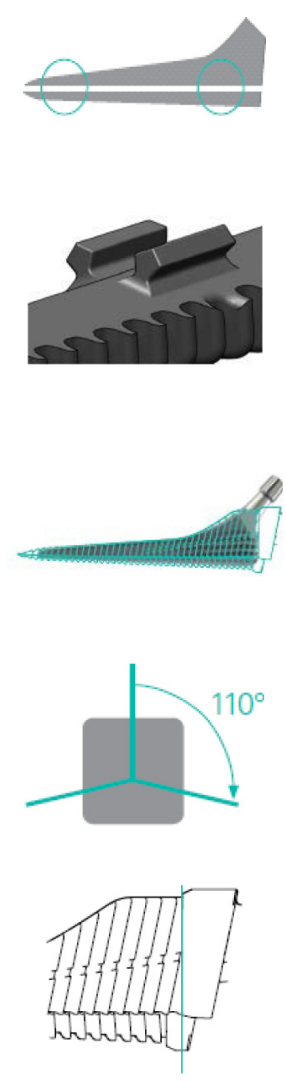


\section{Downward displacement}

The transversal axis of the rasp should be positioned slightly offcenter underneath the motor axis to give the surgeon an optimal view of the operative site.

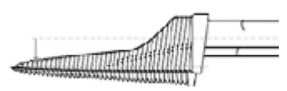

\section{Chips transport}

To better transport the liquid containing the chips to the outside, a kind of 'groove' is created.

Alignment of the saw teeth

All teeth are $12^{\circ}$ proximally inclined. As with a knife, the cutting edge should not be straight.

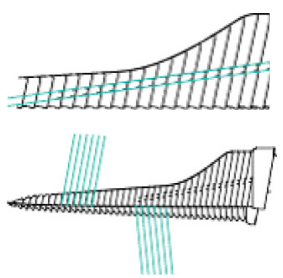

\section{Distance and number of saw teeth}

The distance between the saw teeth is $4 \mathrm{~mm}$ (the stroke path should therefore not exceed $5 \mathrm{~mm}$ ). Each of the two upper tips of the rasp

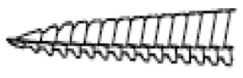
has 38 teeth, while the lower have 44 teeth.

2.5.3 2 Drive. Basically, either a rechargeable battery drive or a pneumatic drive unit can be used. With compressed air, there are several versions available: The decision was made in favor of a pneumatic vane motor for the following reasons:

- Fewer components: Less space required due to compact design with high torque levels. This makes the device smaller and lighter.

- Simpler design: No voltage protection. Fewer weak spots. Better performance: In general, vane motors can achieve better performance and higher speed than DC motors of the same size.

- No cooling: High-performance devices with long operating time need cooling, which is simultaneously provided by the drive unit. It is difficult to implement a battery-driven handheld device of the same performance and without cooling.

- Easy maintenance: Compressed air engines are maintenance free and robust.

- Simple sterilization: A key requirement, which is much more complicated with an electric drive.

2.5.3 3 Transmission. The transmission converts the rotational motion of the motor into the linear motion of the drill bits. The use of an eccentric (inclined rotating disc) provides an elegant technical solution for this (see Fig. 7).

The greater the inclination angle of the disc, the longer the stroke and the more friction at the connecting point between the disc and the drill bits. The larger the diameter of the disc, the longer is the stroke path. However, it requires more power to rotate the disc.

The rotating outer disc of the bearing moves the pins so that the drill tips move synchronously up and down as shown in the motion pattern in Fig. 4.

With a maximum $30^{\circ}$ inclination of the eccentric and a diameter of $21 \mathrm{~mm}$, a maximum stroke of $6 \mathrm{~mm}$ is possible.

2.5.3 4 Connection. The handpiece and the rasp unit are connected by a kind of bayonet mount, as known from a cordless screwdriver or a drilling machine. The connecting element between the compressed air supply at the wall and the handpiece is a dual-chamber hose, which is also connected by a bayonet mount. 

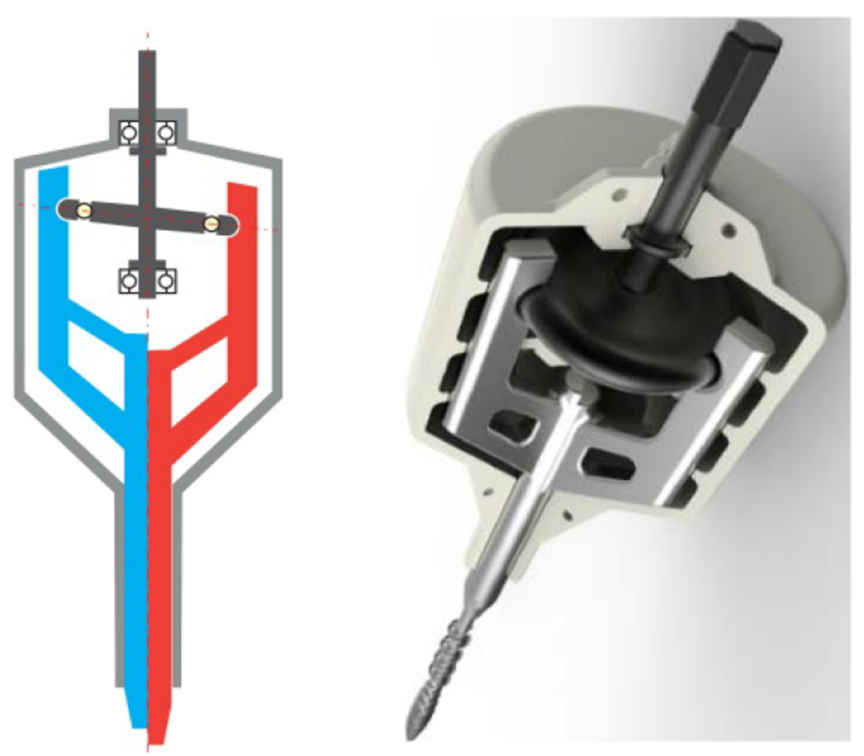

Figure 7: Transmission to convert the rotational motion of the motor into the linear motion of the drill bits.

2.5.3 5 Case/handle. The most important design feature of the external casing is the handle. The design of the handle was the starting point for finding proper forms in the design process. The handle is the interface between the user and the handheld device. The handle provides a direct contact between the user and the hand tool. The handle determines instantly how useful and comfortable the tool is [6].

From surgery pictures and films, we could identify the following typical work position of doctors in hip surgery as shown in Fig. 8a. Six mock-ups of a handle were prepared and tested by different test participants regarding ergonomics (see Fig. 8b). They were asked about the best grip position ('Best way to hold the handle?' and 'Can be operated with one hand?'), the tolerable weight ('How heavy may the device be?'), the proper shape of the handle and how precisely a hole can be drilled in the displayed position.

Two options of the handle were considered suitable. The advantage of the option illustrated in Fig. $8 \mathrm{~d}$ is that it offers a good grip. This is owed to the connection between the gripping area and the housing shaft. Edges extending to the rear provide more contact surface for the hand and more stability.

The option appearing in Fig. 8c convinces by its large gripping area. A handle with a greater inclination angle has no clearly defined gripping area or position. That means you can hold the handle as you like, thus giving more freedom to the surgeon.

In the end, the last option was chosen for implementation. It is to be equipped with a rotatable handle.

The maximum weight tolerated by test-users is $2 \mathrm{~kg}$. For comparison, a cordless screwdriver including battery weighs approximately $2 \mathrm{~kg}$.

2.5.3 6 ON/OFF switch. Requirements for an ON/OFF switch of a surgical instrument are:

- The switch must be possible to access from different gripping positions.

- The switch must be a simple and intuitive operation without effort.

- It must be possible to use the device 'blindly' and the device must not distract the surgeon. 


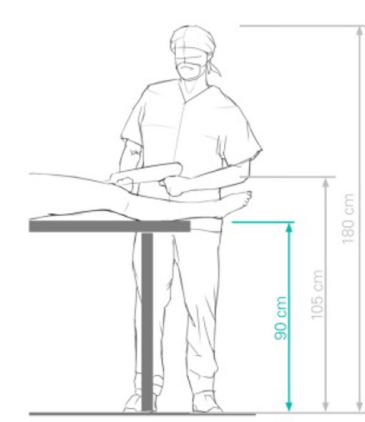

a)

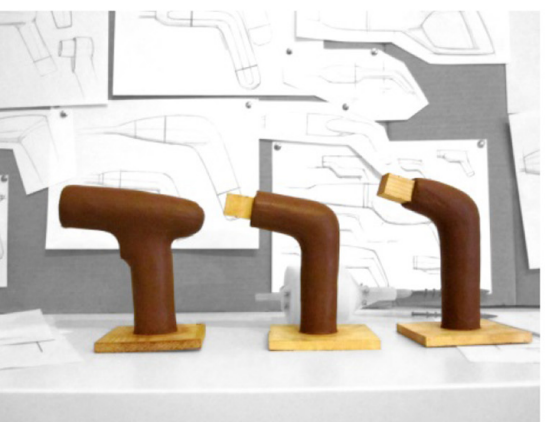

b)

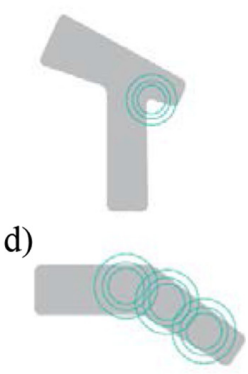

c)

Figure 8: (a) Typical posture of a doctor during a THRP surgery, (b) various mock-ups of the casing and the handle and (c) short-listed design options (the circle indicates where to hold the handle?

A simple and logic switch that can be used for intuitive operation is a push button. The only function of the ON/OFF switch will be to turn the device on and off.

\subsection{The form-finding process}

Functionality and safety are paramount for all products and devices in orthopedic surgery. This is strongly reflected in the external features and the physical effects of the design language of the instruments. In summary, it may be said that the products in orthopedic surgery do not have an appearance that is 'nice' and 'soothing'. In spite of this, or because of it, they hold great potential to create an entirely new and attractive product by selectively changing the design. Additionally, we want to introduce a design vocabulary for biomimetic design in medical products.

\subsubsection{External design}

A brainstorming session was held to define the attributes associated with a surgical instrument before trying to implement them by choosing the right design, materials and colors.

'Clean': Involves a sense of 'cleanliness' and a 'clean' design language: metallic-polished or matt surfaces combined within soft porcelain-type surfaces.

'Simple': A soft, minimalist and reduced design language.

'Hard and cold': Since most instruments are made of metal, they appear to be 'cold' and 'sterile'. Colors are rarely used or only for marking or labeling, to facilitate operation, or enable rapid identification.

'Soft/warm': White - organic.

Essential: Definition by Lovegrove [7] 'Essentialism is about designing things more organically in the future, less constructed in a physical sense, grown rather than constructed...'.

\subsection{Illustrations of the prototype and design model Sirex ${ }^{\mathrm{TM}}$}

\subsubsection{Technical specifications}

- Total weight: $1.5 \mathrm{~kg}$.

- Weight of rasp unit: $700 \mathrm{~g}$. 
- Weight of handpiece: $600 \mathrm{~g}$.

- Drive with compressed air: 7-8 bar.

- Total stroke: $4.5 \mathrm{~mm}$.

- Frequency: 50-70 Hz.

\subsubsection{Views of Sirex ${ }^{\mathrm{TM}}$}

The center of gravity (A) of SIREX lies exactly in the middle. The uniform distribution of weight ensures a good grip.

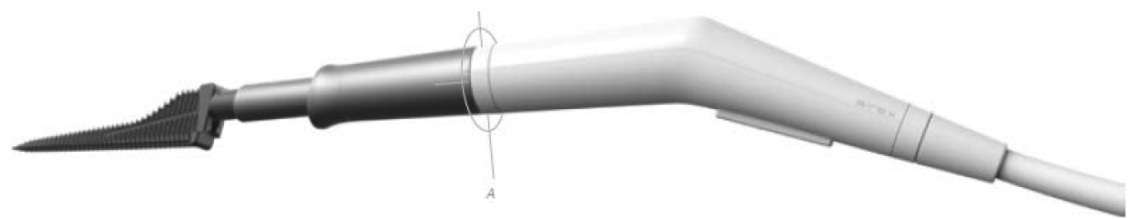

\subsubsection{Function}

Removes cancellous bone from thigh bone after femoral neck has been severed. Then, the prosthesis stem is inserted in a form-fitting manner.

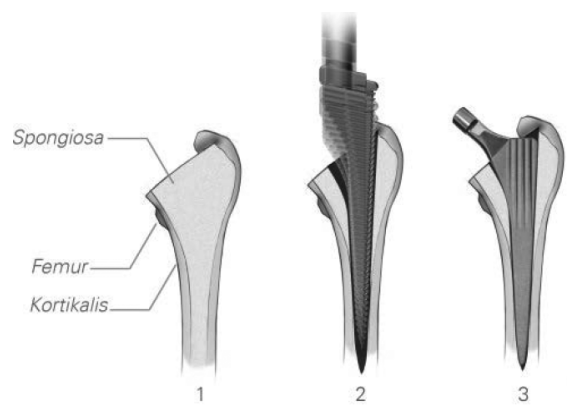

\subsubsection{Hand position}

SIREX can be comfortably held with two hands. The handle at the handpiece is designed to hold it in a way the user finds most comfortable. The front part of the gripping area is equipped with a safety edge, which prevents slipping.
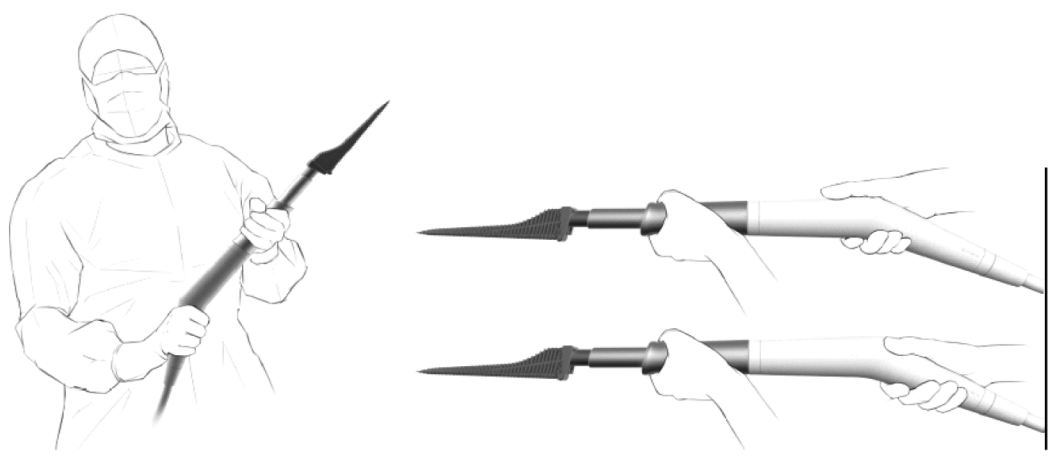


\subsubsection{Changing the rasp unit}

By pressing the button, the rasp unit can be easily and quickly replaced by another size. It is probably not necessary to exchange the unit during surgery, because the size of the prosthesis is selected to match the size of the bone, and the size of the rasp is chosen to match the size of the prosthesis.

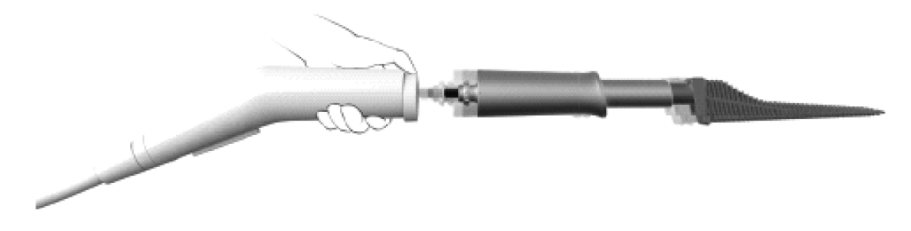

2.7.6 Disassembling the rasp for sterilization

To enable the thorough cleaning and sterilization of the three-part rasp, it is taken apart in a few steps. To do so, the guide tube is rotated by $40^{\circ}$.

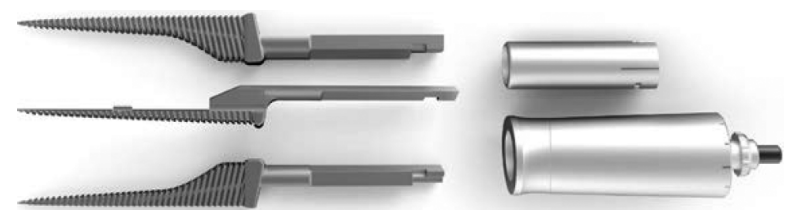

2.7.7 Internal structure

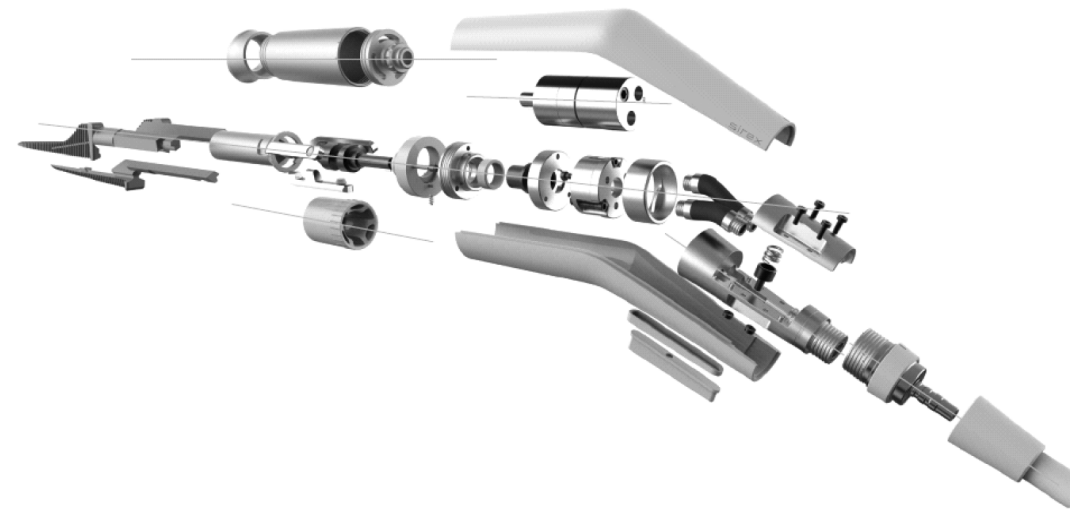

\subsection{Conclusion}

Starting points were the disbelief that there is only one way of boring, i.e. rotary drilling, and the fascination with hymenopterous insects that are able to drill several inches into fresh wood with an egg-laying organ (ovipositor) as thin as a hair, to deposit their eggs.

Beginning from a use case in the field of orthopedic surgery, we followed the top-down process of bionic development (VDI 6220). Analyzing the anatomy and physiology of hymenoptera and some tests of a functional model led to the realization that it is of utmost importance to have the drill composed of three parts to enable a balanced drilling process without any transfer of torque to the work piece. This principle was then implemented in a prototypical functional and design model of a drill rasp for creating cavities in the thigh bones for the form-fitted insertion of cementless hip prosthesis 
stems. Before developing the bionic boring instrument, we tried to get all possible requirements from possible stakeholders and regulations and ergonomic aspects influencing the function, design, materials and color of this bionic medical device. The result was a pneumatic-driven instrument of $1.5 \mathrm{~kg}$ weight with the brand name Sirex ${ }^{\mathrm{TM}}$ in accordance with a wood wasp genus.

Advantages of SIREX ${ }^{\mathrm{TM}}$ are:

Less risk and more security

The 'self-digging', three-part rasp reduces the axial force on the femur.

This unique and bone-friendly rasp reduces the risk of bone damages and fractures during and after surgery.

\section{Direct feedback}

The straight shape of SIREX transfers even the smallest vibration changes in the rasp directly to the hands. In addition, the rasping process itself creates an acoustic feedback for the surgeon, enabling $\mathrm{him} /$ her to immediately recognize the difference in the bone strength (the outer 'Kompacta' is harder than the embedded 'Spongiosa').

Simple and intuitive operation

SIREX is easy to use and its specific shaped handle with large inclination angle allows holding it the way it feels most comfortable to the surgeon.

Easy-to-sterilize

The rasp unit can be disassembled quickly and is easy to clean and sterilize.

There are no screws or edges on the external casing, where dirt particles could settle.

Easy-to-maintain and long life

Due to the modular design, individual components can be separately exchanged. This guarantees a long service life of the device.

For both types of prosthesis systems

The SIREX rasp has been designed for a cementless prosthesis system. Its principle can also be applied to cemented systems where the need of accurate drilling is not so acute (oder: urgent/great).

\section{Improved healing}

Compared with other methods, such as the use of a manual rasp and a hammer, the three-part SIREX rasp creates a better surface structure on the inner wall of the femur. The prosthesis stem is thus better connected to the bone.

\section{REFERENCES}

[1] Rechenberg, R., Die 7 Denkschritte der Bionik, LectureofBiomimetik I. Technische Universität Berlin, available at www.bionik.tu-berlin.de/institut/skript/vorlb1.htm.

[2] Belshaw, R., Grafen, A. \& Quicke, D.L.J., Inferring life history from ovipositor morphology in parasitoid wasps using phylogenetic regression and discriminant analysis. Zoological Journal of the Linnean Society, 139, pp. 213-228, 2003. doi: http://dx.doi.org/10.1046/j.10963642.2003.00078.x

[3] Gao, Y., Ellery, A., Jaddou, M., Vincent, J. \& Eckersley, S., A novel penetration system for in situ astrobiological studies. International Journal of Advanced Robotic Systems, 2(4), pp. 281-286, 2005. doi: http://dx.doi.org/10.5772/5779 
[4] Young Ko, S., Davies, B.L. \& Baena, F.R., Two-dimensional needle steering with a 'Programmable Bevel' inspired by nature: modeling preliminaries. The 2010 IEEE/RSJ International Conference on Intelligent Robots and Systems, October 18-22, Taipei, Taiwan, pp. 2319-2324, 2010. doi: http://dx.doi.org/10.1109/iros.2010.5650859

[5] Neumann, H.W., Mahlfeld, K. \& Lieske, S., Zementiert oder zementfrei - Medizinische und/ oder ökonomische Aspekte? Conference Presentation, p. 8, available at http://www.amou. de/2006/vortraege2006/AMOU2006_Neumann.pdf.

[6] Lindqvist, B., Ergonomie bei Handwerkzeugen-Beurteilung von Kraftwerkzeugen, ed. Atlas Copco, Essen, 1997, available at http://www.zfa-online.de/informationen/leser/volltexte/2003 12003_02_Good_Practice_volltexte/riedel.pdf.

[7] Lovegrove, R., Design Handbook KonzepteMaterialien Stile, eds. Charlotte \& Peter Fiell, ICON Taschen, Köln, 2006. 